forward we will seek to better understand. One explanation is that recording and posting the sessions on YouTube allowed participants to review content asynchronously at a time and location convenient to them, which may have deterred people from attending in person. Following the popularity of this program, future plans are underway to provide an ongoing program of learning in relation to Communication in Science.

3519

\section{Community Engagement And Health Disparities In Clinical And Translational Research Course: A Joint Academic Institution Approach}

Rakale Collins Quarells ${ }^{1}$, Winifred Thompson, $\mathrm{PhD}^{1}$, Elleen Yancey, $\mathrm{PhD}^{1}$ and Tabia Akintobi, $\mathrm{PhD}^{1}$

${ }^{1}$ Morehouse School of Medicine

OBJECTIVES/SPECIFIC AIMS: Current translational research moves beyond bench to bedside and includes translating scientific evidence to clinical practice and into the community settings (T1-T5). This progression is dynamic, involving patient-physician, community, and academic organizational structures and translational strategies. However, basic and clinician scientists are often unprepared and/or ill equipped to successfully conduct community-engaged research which may aid in more efficient translation of their research findings. The recognized need for such training was the impetus for our course which was originally designed and implemented through the innovative and sustainable joint academic-community partnerships of Morehouse School of Medicine and Emory University with the support of Georgia Institute of Technology. Since that time the course has evolved with the recently added partner, University of Georgia. METHODS/STUDY POPULATION: Initially developed and implemented in 2008, the course continues through the Georgia Clinical and Translational Science Alliance, Community Engagement and Research Program (GaCTSA/CERP), a Clinical Translational Science Award (CTSA) (UL1TR002378). The course is an introduction to community-engaged research concepts/methods. This includes behavioral science; community engagement principles; clinical translational research partnerships; and strategies in planning, implementing, evaluating, and disseminating community-engaged research to address health disparities. The course is open to the four GaCTSA academic institutions' faculty, MD, $\mathrm{PhD}, \mathrm{MS}$ in Clinical Research, and the Graduate Certificate in Translational Science students. RESULTS/ ANTICIPATED RESULTS: Students received scholarly and handson training in community engaged research through faculty- and community member-led didactic lectures/interactions, team science activities, and a final assignment involving work with a community-based organization. From 2008-2017 over 230 students have matriculated through this course and many are now involved in community-engaged translational research. Most students in the class were $\mathrm{MD} / \mathrm{PhD}$ students $(33 \%)$, however $21 \%$ were junior faculty, attending physicians (21\%), or fellows/residents/ postdocs (15\%). Evaluations over the years indicate that most students were unware of Community-Based Participatory or community-engagement strategies for conducting translational research. DISCUSSION/ SIGNIFICANCE OF IMPACT: Effective application of community-engaged translational research requires essential skills training to facilitate the translational research paradigm. Translational researchers, at any stage, will benefit from understanding the entire translational research process and the importance of quickly bringing research advances to patients and the community.
3561

\section{Creating a Scientific Community for Outcomes Researchers}

Rebecca Avery Reamey ${ }^{1}$ and Michael J Mugavero

${ }^{1}$ University of Alabama at Birmingham

OBJECTIVES/SPECIFIC AIMS: 1. Foster a community of scholars by centering on training and professional development programs that assist with career progression. 2. Improve heath equity for the community - by developing innovative ways to capture data, address problems, deliver solutions, and disseminate results to patients and people everywhere. 3. Facilitate interdisciplinary teams at all career stages - by providing opportunities for researchers, regardless of discipline and career stage, to engage in dialogue with others. SCOR has developed a series of programs to address each stage in the career arch. LEAD: Learn Enhance Advance Drive develops a pipeline of future leaders within UAB by engaging junior faculty and staff who are in leadership roles or will be in leadership roles soon to develop fundamental competencies. The one-year, cohort-based program enables junior faculty and staff to enhance their interpersonal skills, professional skills, and leadership skills. The program uses the Leadership Competency Model to provide the framework for the chosen topics. The K2R program provides structured activities over a 4- 5 month timeline to assist cohorts of scholars in their preparation of a specific grant application. Leveraging existing programs, like Project Panels, and offerings including a Specific Aims Workshop, K and R Writing Groups, and Mock Study Section, the SCOR extends the engagement of scholars in their ongoing research development. METHODS/STUDY POPULATION: Using quantitative methods, we have conducted surveys to measure effectiveness of weekly didactic sessions and topics. We use qualitative methods such as interviews and focus groups to better understand the relevance of the individual programs and the larger community of SCOR. RESULTS/ANTICIPATED RESULTS: N/A DISCUSSION/ SIGNIFICANCE OF IMPACT: Creating a community of interdisciplinary investigators will greatly impact research at the institution.

3509

\section{Developing a Leadership Alumni Forum to foster a culture of leadership at Mount Sinai}

Janice Lynn Gabrilove, MD, FACP ${ }^{1}$, Layla Fattah ${ }^{1}$, Lisa Bloom and Cara Della Ventura

${ }^{1}$ Mount Sinai School of Medicine

OBJECTIVES/SPECIFIC AIMS: Leadership is an essential and recognized team science competency. To support the development of leadership skills at Mount Sinai, the LEAD (Leadership Emerging in Academic Departments) program, launched in 2016, delivers a structured 12-month blended learning program for junior faculty. The program aims to promote personal and professional leadership capacity, skills and behaviors. Following a competitive application process, 24 participants each year are selected to participate. In its second year, the challenge for the LEAD program leadership is to support alumni in fostering a culture of leadership that extends beyond the 12-month program. In order to promote a leadership community of practice and offer continued support to junior faculty, the LEAD Alumni program aims to bring former LEAD participants together to maintain motivation, share challenges and successes, meet with mentors and role models, and foster an ongoing community of practice that seeks to embed evidenced-based leadership 